-Communication-

\title{
Multi-Channel Optical Monitoring of Neural Signals from Aplysia Ganglion
}

\author{
Michio NAKAShimA*, Satoru ShIONO, Satoshi YAMADA and Mitsuo MAEDA
}

Received August 1, 1988 ; Accepted October 24, 1988

\section{INTRODUCTION}

Generally biological information processing by the neural network requires cooperative activites of a great many neurons. Microelectrode recordings are limited to measuring signals from a small number of neurons simultaneously". In order to monitor a significantly large number of neurons was developed the multi-channel optical monitoring method'. The method utilizes a membrane voltage sensitive dye and photodiode (PD) array detection. It is a new and powerful method to study the signal processing mechanism in the neural network. The first application to the nervous system of Aplysia californica, a sea mollusc, was reported by cohen et al." It is of importance to do optical experiments under various conditions to have more light on the neural information processing mechanism. Here we describe experimental results by applying the optical method to an Aplysia ganglion.

\section{EXPERIMENTAL}

A semi-intact abdominal ganglion with siphon and gill was prepared by dissecting ca. $20 \mathrm{~g}$ Aplysia. The preparation was kept in the chamber filled with sea water as shown in Fig. 1. Abdominal ganglion (AG) was fixed with pins to restrict its movement. The siphon and gill remained intact, since weak touch to the siphon elicited a response of gill contraction.

We adopted experimental procedures according to the cohen's report. Briefly, a voltage sensitive oxonol dye, NK3041 (Nippon

Central Research Laboratory, Mitsubishi Electric Corporation, Anagasaki, Hyogo 661, Japan.

Key words: Optical Monitoring, Neuron, voltage Sensitive Dye
Kankoh-Shikiso, Okayama, Japan), was used because it was shown to give relatively large signals with minimal pharmacological effects. The abdominal ganglion was stained for $20 \mathrm{~min}$ with $0.3 \mathrm{mg} / \mathrm{ml}$ of $\mathrm{NK} 3041$.

An optical monitoring apparatus was homemade, putting a $10 \times 10 \mathrm{PD}$ array on the focusing plane of a microscopé. The light source was a $24 \mathrm{~V}, 300 \mathrm{~W}$ tungsten filament lamp and the lamp output was passed through an interference filter (wave-length, 705+34 nm). We chose the objective magnification of $10 x$ to monitor signals from the whole area of the ganglion (Fig. 1). One PD of the array covered area of $150 \times 150 \mu \mathrm{m}$.
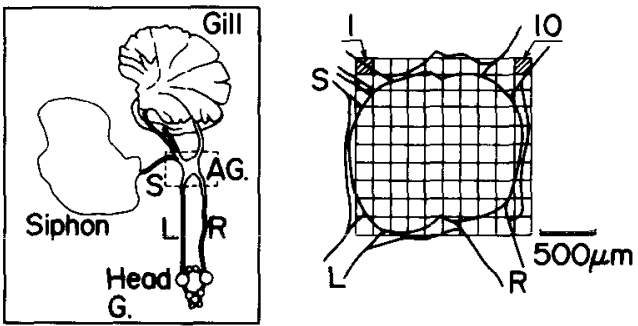

Fig. 1. Schematic diagram of the isolated preparation.

\section{RESULTS AND DISCUSSION}

\section{1 optical recondings of action potentials}

A $60 \mathrm{~s}$ optical monitoring experiment was done with six electrical stimulations to the siphon skin la single pulse of $100 \mathrm{~ms}$ duration at $10 \mathrm{~s}$ interval). Figure 2 shows the 100 channel optical recordings before and after the first stimulation (see the arrow of PD 84). The numbers at the upper and left portion of Fig. 2 indicate positions of the PD elements. More than ten PD's detect action potentials, as observed as spikes, from corresponding neurons. Most of the signals seen on those detectors resulted 
apparently from relatively large neurons. The distribution of the active neurons was not localized. The neuron monitored by PD 84 gives a series of action potentials with the highest frequency after the stimulation. The expanded trace of $\mathrm{PD} 84$ is depicted in the inset. Artifacts, presumably arising from the muscle contraction of the ganglion surface, are also seen in some of the lower and left traces.

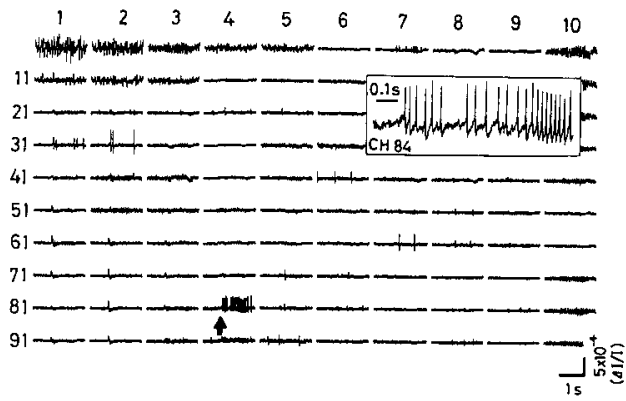

Fig. 2. 100 channel optical recordings for $2 \mathrm{~s}$. Scale bar: vertical, $5 \times 10^{-4}$; horizontal, $1 \mathrm{~s}$.

\subsection{Raster diagram}

Figure 3 shows the raster diagram of action potentials observed in the $60 \mathrm{~s}$ optical monitorings. Each vertical line of the raster represents one action potential. The number to the left of each trace denotes the identified individual neuron, which is obtained by comparing the signals on adjacent detectors. The first two digits of the numbers correspond to the position of the PD's. As a whole, 42 active neurons are detected optically. Each neuron gives differnt action potential pattern after the six stimulations (their timing is shown in the first raster labelled STIM). The active neurons can be classified into three groups; after a stimulation (1) 15 neurons show increasing firing rate (A in Fig. 3), (2) 9 neurons decreasing rate( $B)$, and (3) 18 neurons seem to have no change (C).

\subsection{Number of neurons recorded optically}

The abdominal ganglion contains approximately 1000 neurons. We discriminated only 42 active neurons in this experiment. Alternatively Cohen et al. reported that about 100 active neurons were detected by doing optical recordings upon a quarter part of an abdominal ganglion. The 4-times smaller

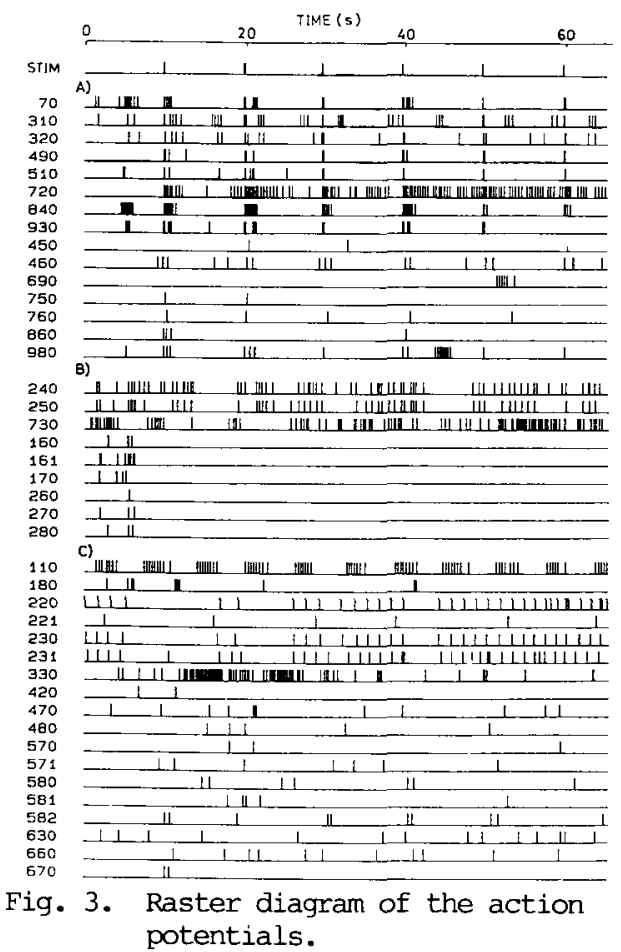

area covered by one PD in their experiment, giving larger signal-to-noise ratio to optical signals from relatively small neurons, can account for the bigger number of the active neurons detected.

The multi-channel optical monitoring method allows to observe spatio-temporally dynamical behavior of the neural network. We will continue optical experiments under various conditions, while improving the optical apparatus, such as installing a PD array with a larger number of PD elements.

Acknowledgment

We are grateful to Drs. Cohen and Kamino for their helpful suggestions.

\section{REFERENCES:}

1) J.A.London, D. Zecevic and L.B.Cohen, J. Neurosci., 7,649 (1987)

2) A.Hotta, K.Kamino, H.Komuro and T.Sakai, J. Physiol., 383,711 (1987)

3) B.M.Salzberg, A.Grinvald, L.B.Cohen, H.V.Davila and W.N.Ross, J. Neurophysiol., 40,1281 (1977)

4) J.-Y.Wu, J.A.London, D.Zecevic, H.P.Hopp, L.B. Cohen and X.Chun, Experientia, 44,369 (1988) 